\title{
Multifunctional lipid-based nanocarriers with antibacterial and anti-inflammatory activities for treating MRSA bacteremia in mice
}

\author{
Chia-Chih Liao ${ }^{1,2 \dagger}$, Huang-Ping Yu ${ }^{1,2 \dagger}$, Shih-Chun Yang ${ }^{3}$, Ahmed Alalaiwe ${ }^{4}$, You-Shan Dai ${ }^{5}$, Fu-Chao Liu ${ }^{1,2^{*}}$ \\ and Jia-You Fang ${ }^{1,5,6^{*}}$ (D)
}

\begin{abstract}
Background: Bacteremia-induced sepsis is a leading cause of mortality in intensive care units. To control a bacterial infection, an immune response is required, but this response might contribute to organ failure. Kidneys are one of the main organs affected by bacteremia. Combination therapies with antibacterial and anti-inflammatory effects may be beneficial in treating bacteremia. This study aimed to develop nanostructured lipid carriers (NLCs) loaded with ciprofloxacin and rolipram that exert a combination of anti-methicillin-resistant Staphylococcus aureus (MRSA) and antiinflammatory effects. Retinol was incorporated into the nanoparticles to transport retinol-binding protein 4 (RBP4) to the kidneys, which abundantly express RBP receptors. The NLCs were fabricated by high-shear homogenization and sonication, and neutrophils were used as a model to assess their anti-inflammatory effects. Mice were injected with MRSA to establish a model of bacteremia with organ injury.

Results: The mean nanoparticle size and zeta potential of the NLCs were $171 \mathrm{~nm}$ and $-39 \mathrm{mV}$, respectively. Ciprofloxacin $(0.05 \%, \mathrm{w} / \mathrm{v})$ and rolipram $(0.02 \%)$ achieved encapsulation percentages of $88 \%$ and $96 \%$, respectively, in the nanosystems. The minimum bactericidal concentration of free ciprofloxacin against MRSA increased from 1.95 to $15.63 \mathrm{\mu g} / \mathrm{ml}$ when combined with rolipram, indicating a possible drug-drug interaction that reduced the antibacterial effect. Nanoparticle inclusion promoted the anti-MRSA activity of ciprofloxacin according to time-kill curves. The NLCs were found to be largely internalized into neutrophils and exhibited superior superoxide anion inhibition than free drugs. Retinol incorporation into the nanocarriers facilitated their efficient targeting to the kidneys. The NLCS significantly mitigated MRSA burden and elastase distribution in the organs of MRSA-infected animals, and the greatest inhibition was observed in the kidneys. Bacterial clearance and neutrophil infiltration suppression attenuated the bacteremia-induced cytokine overexpression, leading to an improvement in the survival rate from 22\% to 67\%.
\end{abstract}

Conclusions: The dual role of our NLCs endowed them with greater efficacy in treating MRSA bacteremia than that of free drugs.

Keywords: Nanostructured lipid carriers, Ciprofloxacin, Rolipram, Bacteremia, Methicillin-resistant Staphylococcus aureus, Sepsis

*Correspondence: ana5189@adm.cgmh.org.tw; fajy@mail.cgu.edu.tw ${ }^{\dagger}$ Chia-Chih Liao and Huang-Ping Yu contributed equally to this work ${ }^{1}$ Department of Anesthesiology, Chang Gung Memorial Hospital, 5 Fuxing Street, Kweishan, Taoyuan 333, Taiwan

${ }^{5}$ Pharmaceutics Laboratory, Graduate Institute of Natural Products, Chang Gung University, 259 Wen-Hwa 1st Road, Kweishan, Taoyuan 333, Taiwan

Full list of author information is available at the end of the article

\section{Background}

Bacteremia is described as the appearance of pathogenic bacteria in the bloodstream. The most common cause of bacteremia is Staphylococcus aureus infection [1]. In the United States, the annual incidence of $S$. aureus bacteremia has been $4.3-38.2$ per 100,000 persons, with an

c) The Author(s) 2021. This article is licensed under a Creative Commons Attribution 4.0 International License, which permits use, sharing, adaptation, distribution and reproduction in any medium or format, as long as you give appropriate credit to the original author(s) and the source, provide a link to the Creative Commons licence, and indicate if changes were made. The images or other third party material in this article are included in the article's Creative Commons licence, unless indicated otherwise in a credit line to the material. If material is not included in the article's Creative Commons licence and your intended use is not permitted by statutory regulation or exceeds the permitted use, you will need to obtain permission directly from the copyright holder. To view a copy of this licence, visit http://creativeco mmons.org/licenses/by/4.0/. The Creative Commons Public Domain Dedication waiver (http://creativecommons.org/publicdomain/ zero/1.0/) applies to the data made available in this article, unless otherwise stated in a credit line to the data. 
associated mortality of $20 \%$, since the 1990 s [2]. Methicillin-resistant $S$. aureus (MRSA) is an additional bacterial burden with a high death rate. The mortality of patients with critical MRSA bacteremia can exceed 60\% [3]. Individuals with bacteremia usually experience systemic inflammation due to a proinflammatory cytokine burst by immune cells [4]. Bacteremia, followed by acute inflammation, can lead to sepsis and multiple organ failure. Sepsis is an inflammatory and immune response to infection. The activation of proinflammatory mediators by sepsis contributes to organ damage, especially damage to the heart, lungs, and kidneys. The majority of bacteria identified in sepsis patients are gram-positive pathogens, such as $S$. aureus, with an increasing trend in drugresistant microbes [5]. Severe septic shock is observed in $38 \%-40 \%$ of patients with $S$. aureus bacteremia [6].

Despite therapeutic innovation, the mortality rate due to bacteremia-induced sepsis is still high [1, 7]. Resistance to first-line antibiotics complicates the treatment of MRSA bacteremia [8]. The poor clinical outcomes highlight the urgent need for developing improved therapy for MRSA bacteremia. In the last decade, nano-based drug delivery systems have been increasingly used to improve antibacterial and anti-inflammatory treatments. The large surface-to-volume ratios and drug loading capacities of nanoparticles contribute to their biological advantages, namely, increased drug solubility, enhanced drug storage stability, improved bioavailability, prolonged half-life, and efficient organ targeting [9]. Nanoparticulate drug delivery is considered beneficial for the encapsulation of active agents against resistant bacteria and inflammatory disorders [10, 11]. Nanostructured lipid carriers (NLCs) are lipid-based nanocarriers that allow increased drug encapsulation because of the imperfect lipid matrix that is composed of lipids in both solid and liquid forms. The use of biodegradable lipids leads to higher tolerability of NLCs than that of polymeric and metallic nanoparticles [12]. There have has been no investigation on the application of nanomedicine to treat bacteremia. To date, clinical trials aiming to study anti-inflammatory approaches have failed to improve the outcomes of bacteremia-induced sepsis [13]; therefore, the development of novel therapeutic modalities with antibacterial and anti-inflammatory properties remains a critical issue. A new emerging regimen for inhibiting the inflammatory response is currently being investigated for the treatment of bacteremia-induced sepsis [7]. The use of treatments that combine antibiotics and anti-inflammatory therapies has been proven to reduce mortality in sepsis compared to the use of antibiotics alone [14, 15]. Therefore, this study explored the applicability of intravenous NLCs with combined anti-MRSA and anti-inflammatory activities for the treatment of bacteremia. To this end, ciprofloxacin and rolipram were both incorporated into NLCs.

The standard therapy for MRSA bacteremia includes the early intravenous administration of antibiotics, such as vancomycin, daptomycin, and teicoplanin [16]. Ciprofloxacin, a broad-spectrum antibiotic belonging to the class of fluoroquinolones, may also be intravenously administered to treat bacteremia [17-19]. To alleviate the inflammation stimulated by bacteremia or sepsis, phosphodiesterase 4 (PDE4) inhibitors have been proven effective in mitigating the cytokine storm induced by bacteria or viruses for the treatment of sepsis-induced organ damage [20-23]. The inhibition of PDE4 is associated with the suppression of immune overactivity via the prevention of cAMP degradation, as PDE4 can decrease cAMP expression to activate the inflammatory response. One representative PDE4 inhibitor is rolipram, which is a novel PDE4 inhibitor. Rolipram improves cardiac and renal function through the inhibition of cytokine overexpression in a rodent model of sepsis [24, 25]. Neutrophils are the most important cell in the host response to bacterial infection [26], and their migration is a fundamental component of the immune response elicited by bacteremia [12]. In this study, primary human neutrophils were used as model cells to examine the anti-inflammatory effect of NLCs. We established an MRSA-infected bacteremia model in rats to assess the inhibitory effect of NLCs on MRSA burden, acute inflammation, and mortality rate. We also monitored the biodistribution of the nanoparticles in various organs.

\section{Results}

\section{Physicochemical features of NLCs}

The particle size, polydispersity index (PDI), and surface charge are important features of nanocarriers that govern their stability and biological performance. As shown in Table 1, the mean diameter of the prepared lipid-based nanoparticles was $171 \mathrm{~nm}$. The NLCs exhibited monodispersity with a PDI of 0.42 . The zeta potential of the dual drug-loaded nanoparticles was highly negative at -39 $\mathrm{mV}$. The encapsulation efficiency of the drugs determined

Table 1 Physiochemical properties of the nanostructured lipid carriers on nanoparticulate size, polydispersity index (PDI) and zeta potential

\begin{tabular}{lr}
\hline Property & \multicolumn{1}{c}{ Value } \\
\hline Size $(\mathrm{nm})$ & $171.40 \pm 5.82$ \\
Polydispersity index & $0.42 \pm 0.04$ \\
Zeta potential $(\mathrm{mV})$ & $-38.80 \pm 0.61$ \\
\hline
\end{tabular}

Each value represents the mean $\pm \operatorname{SEM}(n=3)$ 
by ultracentrifugation showed that the encapsulation percentages of ciprofloxacin and rolipram in the NLCs were $88.21 \pm 7.0 \%$ and $95.81 \pm 12.24 \%$, respectively. The release of ciprofloxacin and rolipram from the nanocarriers was examined. The obtained profiles of ciprofloxacin release as a function of time indicated an initial burst release from the control vehicle and NLCs (the left panel of Additional file 1: Figure S1). Ciprofloxacin release from the free control reached nearly $100 \%$ after $24 \mathrm{~h}$, indicating a fast release. Inclusion in the nanoparticles decreased the release rate of ciprofloxacin. A similar trend was detected in the case of rolipram (the right panel of Additional file 1: Figure S1).

\section{Anti-MRSA activity of NLCs}

The capability of the NLCs to inhibit MRSA growth was examined to understand their potential for treating MRSA-induced bacteremia. As shown in Table 2, the minimum bactericidal concentration (MBC) of ciprofloxacin alone was $0.98 \mu \mathrm{g} / \mathrm{ml}$. Rolipram alone did not exhibit

Table 2 The MBC $(\mu \mathrm{g} / \mathrm{ml})$ of ciprofloxacin in free form and nanostructured lipid carriers in the presence or absence of rolipram against MRSA

\begin{tabular}{ll}
\hline Formulation & MBC $(\boldsymbol{\mu g} / \mathbf{m l})$ \\
\hline Ciprofloxacin in free form & 0.98 \\
Ciprofloxacin + rolipram & 7.81 \\
Ciprofloxacin in NLCs & $0.98-1.95$ \\
Ciprofloxacin + rolipram in NLCs & 7.81
\end{tabular}

Each value represents the mean $\pm \operatorname{SEM}(n=3)$ any anti-MRSA activity. Surprisingly, treatment with the combination of both drugs significantly reduced the anti-MRSA activity of ciprofloxacin. The MBC of ciprofloxacin increased by 8 -fold after rolipram intervention. The antimicrobial potency of the NLCs loaded with ciprofloxacin was found to have a MBC between 0.98 and $1.95 \mu \mathrm{g} / \mathrm{ml}$. Similar to their free forms, the combination of ciprofloxacin and rolipram in the NLCs increased the MBC of ciprofloxacin by 4-8-fold. Time-kill curves provide information regarding the extent and rate of antiMRSA activity. Figure 1a and $\mathrm{b}$ show the time-response MRSA growth inhibition of ciprofloxacin and rolipram at low $(0.5$ and $0.2 \mu \mathrm{g} / \mathrm{ml})$ and high doses $(1$ and $0.4 \mu \mathrm{g} / \mathrm{ml})$, respectively. The control group was the MRSA population not treated with drugs or nanoparticles. Treatment with rolipram, in both its free and nanoparticulate forms, had no significant influence on MRSA growth compared to the no treatment control. The NLCs without drugs also showed no MRSA inhibition; however, the formulations containing ciprofloxacin markedly suppressed the growth of MRSA. The ciprofloxacin-loaded NLCs were more efficient than free ciprofloxacin in inhibiting bacterial growth. At high doses, free and nanoparticulate ciprofloxacin completely inhibited MRSA growth over $24 \mathrm{~h}$. However, a combination of ciprofloxacin and rolipram in free form weakened the anti-MRSA effect.

\section{Anti-inflammatory activity of NLCs}

Primary neutrophils were used as model cells to examine the anti-inflammatory activity of the NLCs and to confirm the possible activity that mitigates bacteremiaelicited acute inflammation. Prior to assessing inflammation inhibition, a cytotoxicity experiment was performed
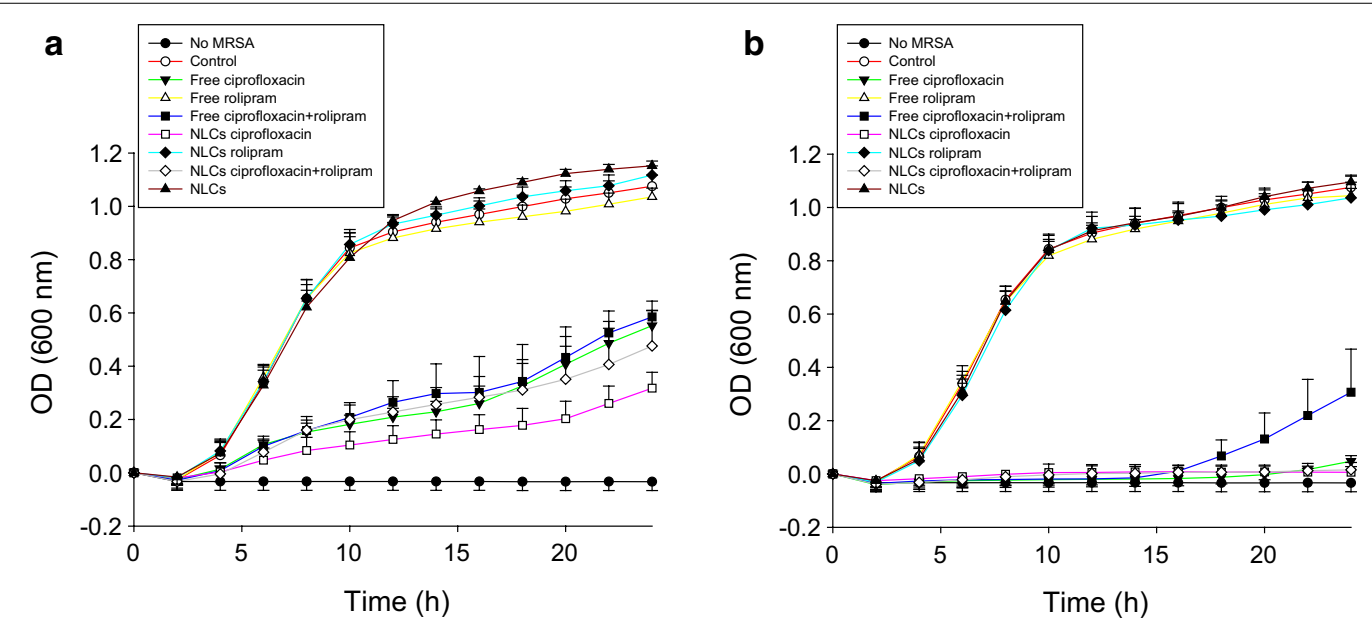

Fig. 1 Determination of the antibacterial activity of free drugs and NLCs against planktonic drug-resistant MRSA: a the time-killing curves of ciprofloxacin $(0.5 \mu \mathrm{g} / \mathrm{ml})$ and rolipram $(0.2 \mu \mathrm{g} / \mathrm{ml})$; and $\mathbf{b}$ the time-killing curves of ciprofloxacin $(1 \mu \mathrm{g} / \mathrm{ml})$ and rolipram $(0.4 \mu \mathrm{g} / \mathrm{ml})$. All data are expressed as the mean \pm SEM ( $n=3)$. MRSA, methicillin-resistant Staphylococcus aureus; OD, optical density; SEM, standard error of mean 
by measuring lactate dehydrogenase (LDH) leakage, and the results are shown in Fig. 2a. Various concentrations of ciprofloxacin $(0.75-75 \mathrm{nM})$ and/or rolipram $(0.3-30$ $\mathrm{nM}$ ) in both free and nanoparticulate forms did not exert cytotoxic effects on neutrophils. Activated neutrophils produce superoxide anions to induce oxidative stress. We observed that ciprofloxacin, in both its free and nanoparticulate forms, failed to suppress extracellular superoxide anion production (Fig. 2b). Rolipram inhibited superoxide anion production in a concentration-dependent manner, and the nanocarrier formulation $(59 \%-88 \%$ suppression) showed greater inhibition than the free drug (17\%-78\% suppression). The inhibition of oxidative stress by rolipram was not a consequence of cytotoxicity since there was no LDH leakage after rolipram treatment. In fact, the incorporation of ciprofloxacin decreased the rolipram-induced superoxide inhibition. Free ciprofloxacin even completely prevented the inhibitory activity of free rolipram.

We next used confocal microscopy to visualize the uptake of the NLCs by human neutrophils. The nuclei were stained with DAPI and emitted a blue signal, as shown in Fig. 2c. Neutrophils treated with low $(5 \mu \mathrm{l})$ and high $(10 \mu \mathrm{l})$ amounts of NLCs emitted increased red fluorescence compared to the nontreated neutrophils. The lipid nanoparticles were predominantly located in the cytoplasm as punctate dots. The distribution of the red fluorescence was comparable between the neutrophils treated with low and high amounts of NLCs. As shown in Fig. 2d, the intracellular fluorescence, as analyzed by flow cytometry, markedly increased by a factor of $2 \log$ after nanoparticle internalization.

\section{Biodistribution of NLCs in rats}

The distribution of NLCs after injection into the human body is important for understanding possible drug deposition in organs and for achieving effective therapy. To assess the biodistribution of NLCs in peripheral organs after intravenous injection, the lipophilic dye iFluor 790 was loaded into the nanocarriers for near-infrared (NIR) monitoring. We used healthy rats, rather than mice, as the animal model in this biodistribution experiment due to their larger organ size and lower autoNIR signal in organs, which facilitated clearer visualization. Figure 3 shows the qualitative and quantitative analyses of nanoparticle distribution by IVIS. The rats that received no treatment (blank control) exhibited negligible autoNIR signals in their organs, except for the gastrointestinal tract (Fig. 3a). The NIR signal in the organs before and after receiving free dye was comparable (Fig. 3b), indicating a rapid degradation of the dye in circulation. The intravenously injected NLCs were deposited mainly in the kidneys (Fig. 3c). The NIR signal in the other organs after nanoparticle distribution was comparable to that in the blank control group. The renal distribution of nanoparticles can be affected by retinol; therefore, we reduced the retinol concentration in the NLCs from $0.25-0 \%$ and $0.1 \%$ to visualize their biodistribution, and we found that the NIR signal was weaker (Fig. 3d and e). The quantification of the NIR intensity indicated a lower accumulation of NLCs without retinol in the kidney than that of the NLCs with retinol (Fig. 3f). After the intravenous injection of the NLCs with different percentages of retinol, there was no significant difference in the NIR intensity in other organs.

\section{Use of NLCs for MRSA bacteremia treatment}

To evaluate the protective effects of free drugs and NLCs on MRSA-induced bacteremia in mice, we examined the survival rate of the mice four days after intravenous injection of the drug-loaded formulations. The mice infected with MRSA displayed 22\% survival on day four, as shown by the Kaplan-Meier curve in Fig. 4a. Following treatment with the combination of free ciprofloxacin $(2.5 \mathrm{mg} /$ $\mathrm{kg})$ and rolipram $(1 \mathrm{mg} / \mathrm{kg}), 41 \%$ of the infected mice survived after four days of MRSA intervention. We found an improvement in the survival of the bacteremic mice $(67 \%)$ that received NLCs containing $0.25 \%(\mathrm{w} / \mathrm{v})$ retinol, suggesting significant disease remission. The surviving mice were sacrificed to quantify the MRSA accumulation in blood and peripheral organs. No MRSA was detected in the animals that did not receive bacterial injection (control) (Fig. 4b). Four days after injection, MRSA was largely deposited in the blood and organs. The MRSA colony-forming unit (CFU) count was log-transformed in this figure. Neither the free drugs nor the NLCs reduced the MRSA CFU counts in the heart and spleen. Significant inhibition of the MRSA CFU counts in the blood and lungs was observed after treating the mice with the free drugs. The NLCs caused a $1-2 \log$ reduction in the CFU counts in the blood, lungs, liver, and kidneys compared to those in the infected mice without treatment;

(See figure on next page.)

Fig. 2 Effects of free drugs and NLCs on primary human neutrophils ( $6 \times 10^{5}$ cells $\left./ \mathrm{ml}\right)$ : a neutrophil cytotoxicity assay by LDH determination; $\mathbf{b}$ the measurement of extracellular superoxide production; $\mathbf{c}$ the uptake of rhodamine 800-loaded NLCs by human neutrophils observed via confocal microscopy; and $\mathbf{d}$ the fluorescence intensity of rhodamine 800 in the neutrophils analyzed by flow cytometry. All data are expressed as the mean $\pm \operatorname{SEM}(n=3)$. * $p<0.05$ as compared to control group. LDH, lactate dehydrogenase; DAPI, 4,6-diamidino-2-phenylindole 

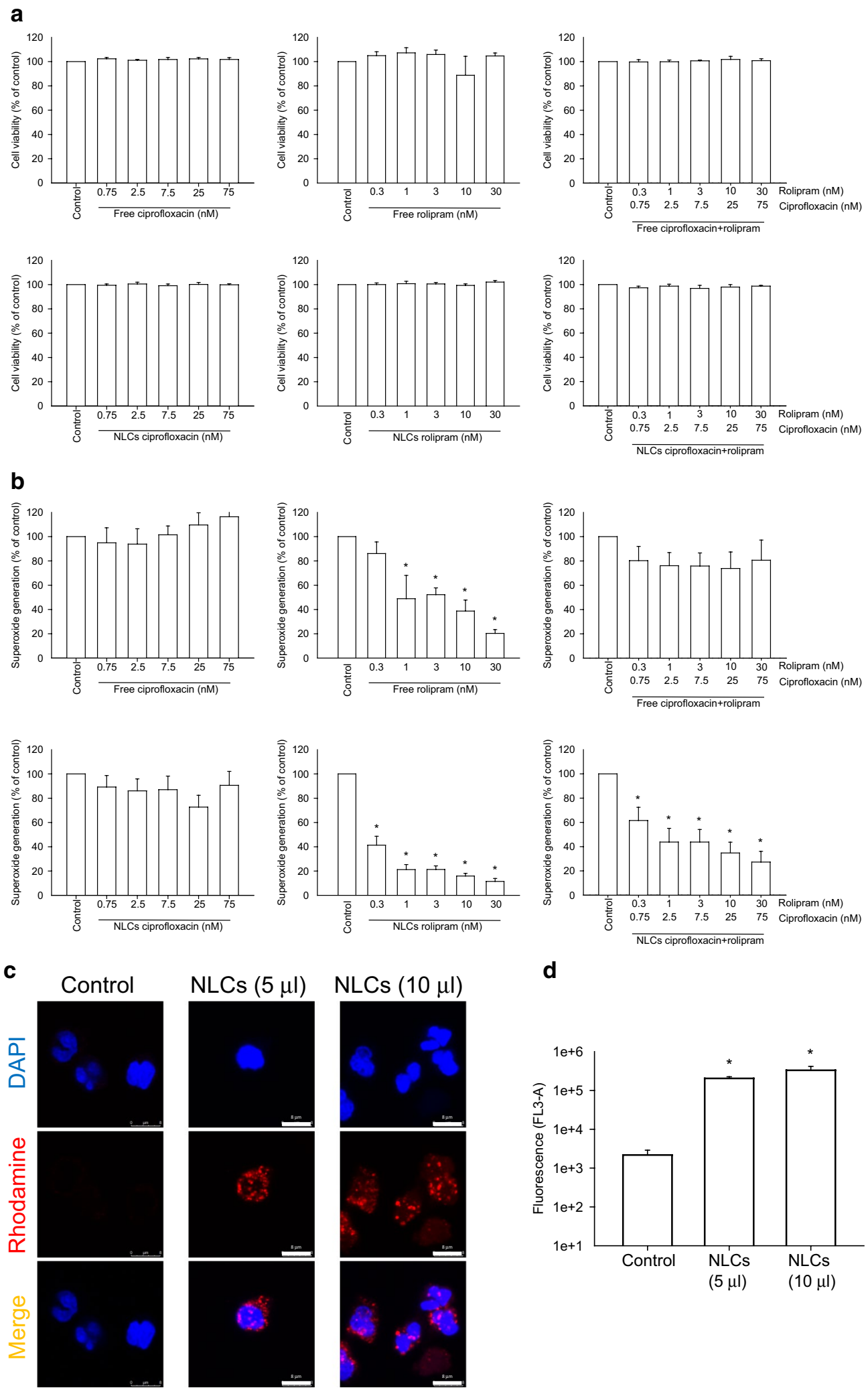
the greatest decrease in the CFU count was observed in the kidneys. Statistical analysis revealed that compared with the free drug control, the nanoparticles led to a significant reduction in MRSA in the liver and kidneys. To explore the anti-inflammatory activity, the excised organs were visualized by ex vivo bioimaging to assess the elastase distribution in organs. Elastase is an indicator of activated and infiltrating neutrophils. As shown in Fig. 4c, the elastase signal (bright red color) in all the organs showed an increasing trend in response to MRSA infection. The administration of the combination of ciprofloxacin and rolipram in their free forms decreased the elastase levels in the heart, but this decrease was not observed in the other organs. We observed that the elastase levels in the heart, lungs, spleen, and kidneys were reduced in the group that received the NLCs, but the NLC-treated group did not exhibited a reduction in the elastase level in the liver.

Only a limited number of mice survived long enough to assess the MRSA burden and elastase levels; therefore, we shortened the treatment duration to $40 \mathrm{~h}$ for further examination. As shown in Fig. 5a, the MRSA inhibition in the heart and lungs was comparable between the free drug and NLC treatment groups. The bacterial count in the blood, liver, and kidneys was significantly reduced by the NLCs compared with the free drugs. There was a $2-\log$ reduction in the MRSA growth in the blood and kidneys in the NLC group compared to the MRSA group without drug intervention. After the four-day treatment, neither the free drugs nor the NLCs decreased the MRSA accumulation in the spleen. The elastase distribution trends in the mice after $40 \mathrm{~h}$ of treatment were consistent with the results of the longer treatment (four days), as shown in Fig. 5b. The NLCs more substantially decreased the elastase levels than the free drugs in the infected mice. Next, we examined whether free drugs or NLCs suppressed proinflammatory cytokine expression in the organs involved in bacteremia. The ELISA results are shown in Fig. $5 \mathrm{c}-\mathrm{g}$. The level of IFN- $\gamma$ in the MRSA-infected animals significantly increased compared with that in the healthy control animals (Fig. 5c). The administration of the combination of ciprofloxacin and rolipram in their free and nanoparticulate forms caused comparable inhibition of IFN- $\gamma$. The administration of NLCs resulted in significantly lower expression of IL- $1 \beta$ in the heart and kidneys (Fig. 5d). This effect was not observed in the group treated with free drugs. Treatment with free drugs and NLCs significantly inhibited the IL- $1 \beta$ overexpression in the lungs and liver to comparable degrees. IL- $1 \beta$ expression in the spleen increased in the free drug-treated mice, but not in the infected mice without drug intervention, compared to the healthy control mice. We could not explain this phenomenon with the existing knowledge. Further study is needed to delineate the possible mechanisms.
MRSA intervention did not have a significant effect on IL-6 expression in the organs, except for the spleen (Fig. 5e). Nevertheless, compared with no drug treatment, the NLCs still exhibited the capability to inhibit IL-6 expression in the lungs and spleen. This result suggests that the protein concentration of IL-17A significantly increased in all organs after MRSA challenge (Fig. 5f). The free drugs were capable of inhibiting IL-17A in the lungs and kidneys. NLC administration further reduced IL-17A production in the lungs, liver, and kidneys by $61 \%, 52 \%$, and $67 \%$, respectively. The NLCs decreased IL-17A production in the kidneys to the baseline levels observed in the controls. No IL-17A suppression was observed in the heart and spleen of infected mice after treatment with the free drugs or NLCs. TNF- $\alpha$ expression in all the organs increased by $2.1-3.7$-fold in the MRSA-infected animals compared to that in the healthy animals (Fig. 5g). The TNF- $\alpha$ levels in the heart and liver were markedly reduced to baseline levels after treatment with both the free drugs and NLCs. The free drugs exerted no effect on the suppression of TNF- $\alpha$ overexpression in the kidneys. Nanocarrier treatment resulted in a 2-fold decrease in the TNF- $\alpha$ levels in the kidneys compared with those observed in the infected group.

\section{Histological observation}

Forty hours after MRSA challenge, the mice were sacrificed to observe the histology of the different organs. The histology of the spleen was excluded in this experiment because of the negligible effects of free drug or nanoparticle treatment on the inhibition of MRSA and the suppression of some cytokines. The hearts of the healthy mice showed intact myocytes and cardiac muscle fibers, as shown in Fig. 6. MRSA infection resulted in a large area of cell debris with suppurative inflammation and immune cell infiltration. The area of suppurative inflammation was reduced after free drug and NLC treatment. Hematoxylin and eosin (H\&E) staining of the lungs of the infected mice showed interstitial congestion with a loss of lung architecture. Edema and immune cell recruitment in the parenchyma were also observed in the MRSA-infected lungs. Moreover, interstitial thickening with congestion was worsened by free drug administration. The NLCs, however, markedly attenuated the MRSA-induced lung injury with a greater region of preserved pulmonary architecture. The hepatocytes in the liver tissues of the normal mice appeared well organized. Congestion and inflammatory cell infiltration were observed in hepatic tissues after MRSA injection. This was a typical observation of liver infection in the early stage. While congestion could be improved by the free drugs and NLCs, this improvement was limited. H\&E staining revealed that the kidney structure consisted mainly of renal tubules and 

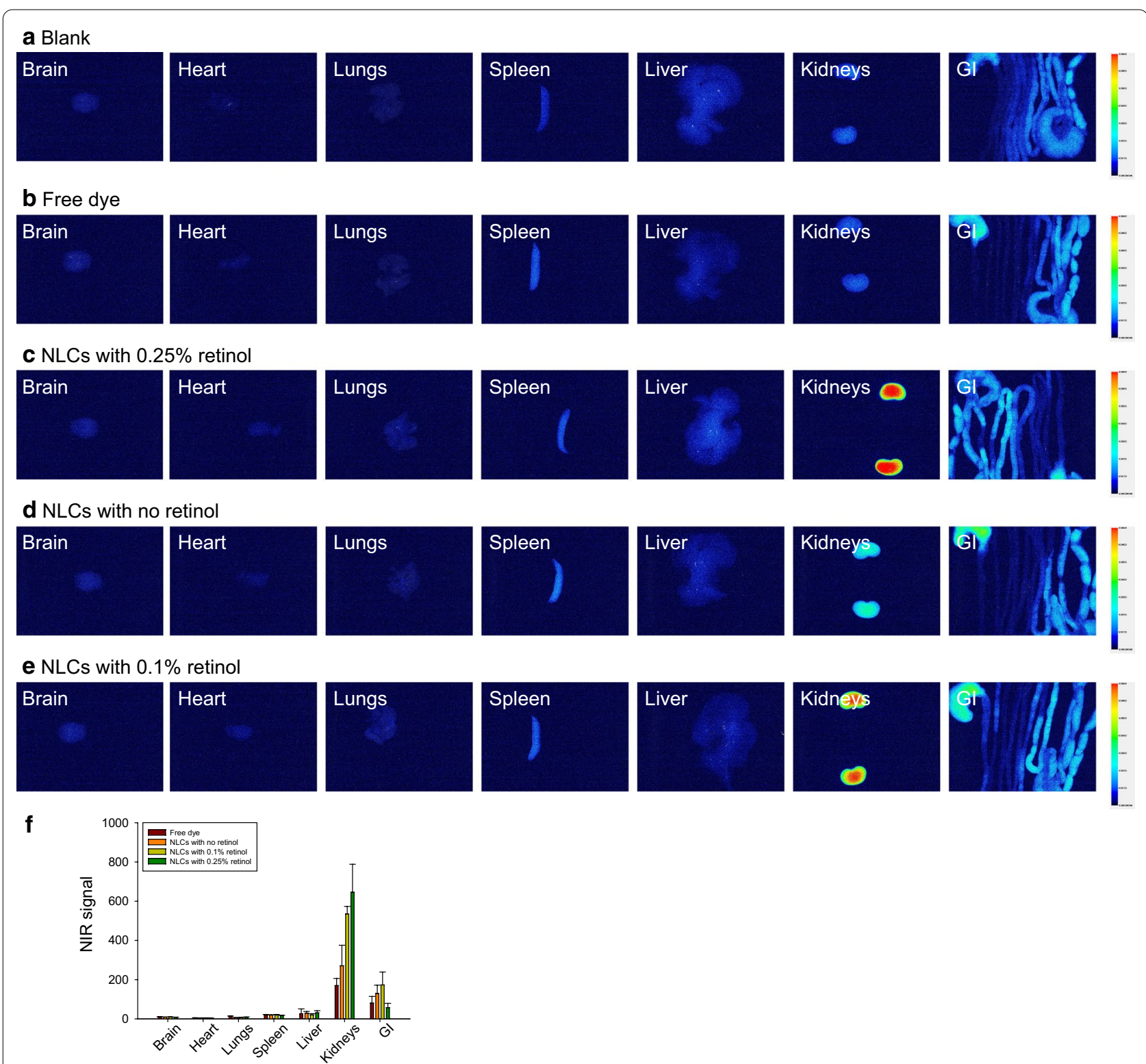

Fig. 3 Ex vivo bioimaging of organs in the rats receiving intravenous iFlour 790-loaded NLCs: a the blank group (non-treated control); b Free iFlour 790; c iFlour 790-loaded NLCs containing 0.25\% retinol; d iFlour 790-loaded NLCs containing 0\% retinol; e iFlour 790-loaded NLCs containing $0.1 \%$ retinol; and $\mathbf{f}$ the NIR intensity of iFlour 790 in the organs. All data are expressed as the mean \pm SEM $(n=6)$. Gl, gastrointestinal tract; NIR, near-infrared

medulla. The renal tissues in the control mice appeared normal and regular. MRSA challenge induced cell debris accumulation and acute suppurative inflammation in the tissue. Following MRSA intervention, immune cell infiltration and tubule necrosis were also visualized. Following MRSA treatment, the kidney structure was preserved by the injection of the drugs in their free forms. Congestion and immune cell recruitment were reduced by the NLCs compared to those observed in the infected group.
The MRSA distribution in the organs was examined using $S$. aureus Rosenbach antibody immunohistochemistry, as shown in Fig. 7a. There were bacterial clusters in the hearts of the MRSA-treated mice. These clusters were limited by the free and nanoparticulate forms of the drugs. The MRSA distribution in the lungs was also inhibited by the free drugs and NLCs. However, the MRSA distribution in the liver could not be clearly observed in all the groups. This could be due to the minimal aggregation of MRSA in the liver, which makes it 

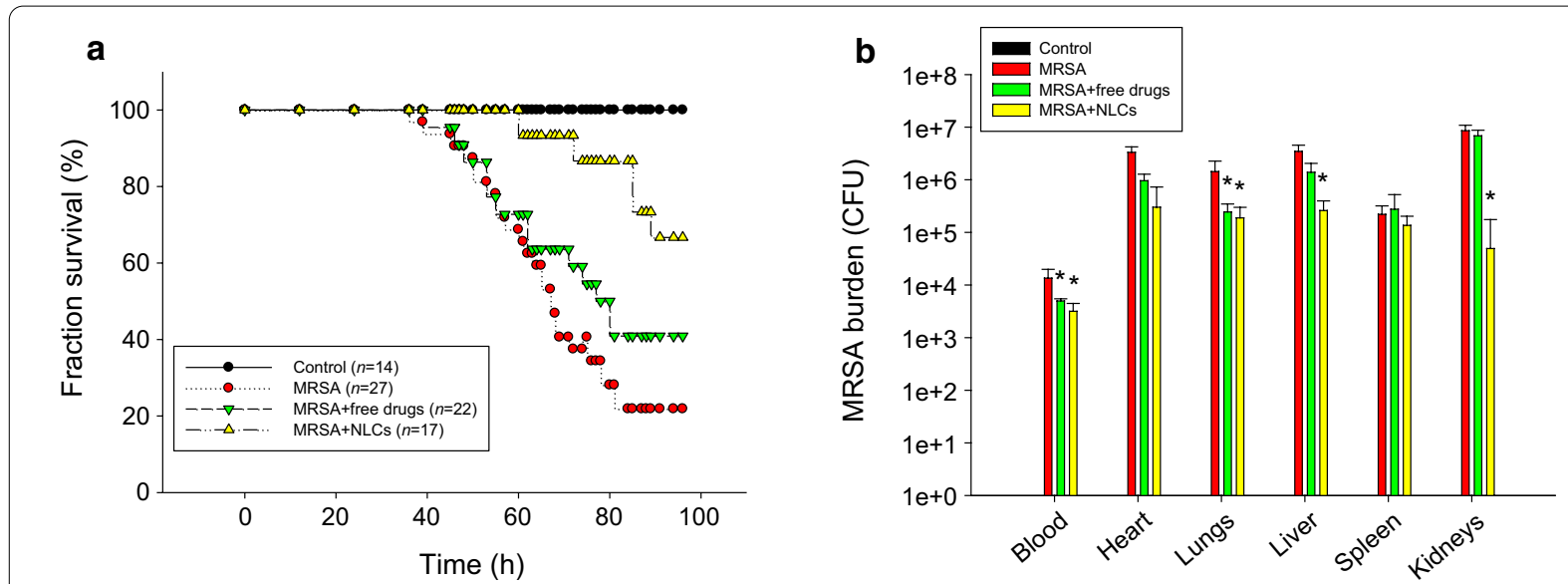

C

Control

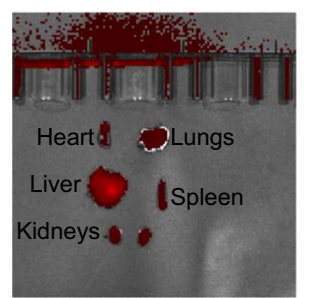

MRSA
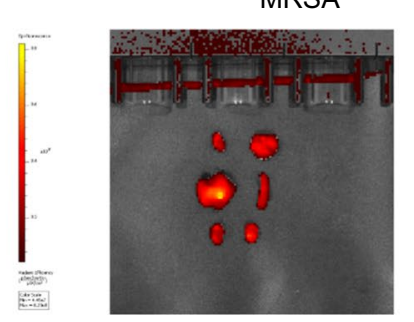

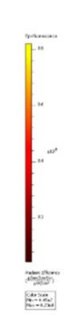

MRSA+free drug

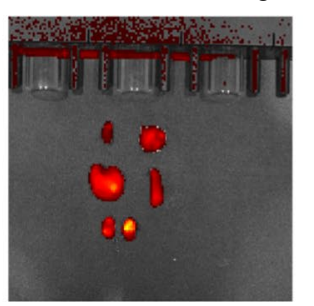

MRSA+NLCs
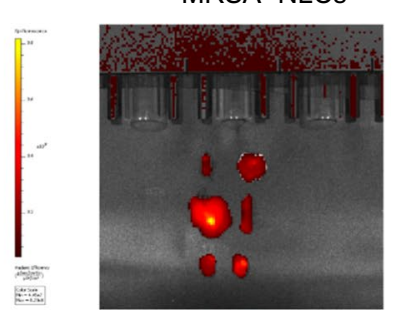

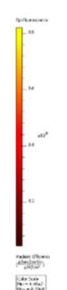

Fig. 4 In vivo effect of free drugs and NLCs on MRSA-infected mice during 4 days: a the survival rate of the mice determined by Kaplan-Meier curves; $\mathbf{b}$ MRSA CFU in organs; and c elastase distribution in organs observed by IVIS. All data are expressed as the mean \pm SEM $(n=14-27$ for survival rate and $n=5$ for MRSA CFU and elastase analysis). CFU, colony-forming unit; MRSA, methicillin-resistant Staphylococcus aureus

difficult to recognize abscesses in the tissue. The MRSA bacterial clusters in the kidneys were reduced by the NLCs but not by the free forms of the drugs. Ly6G is a biomarker of neutrophil migration into organs that is used to characterize the features of sepsis. We visualized neutrophil accumulation in the heart $40 \mathrm{~h}$ after MRSA injection, as shown in Fig. 7b. Neutrophil diffusion was attenuated by the administration of the drugs, and the NLCs demonstrated better performance. On the other hand, the neutrophil infiltration in the lungs and liver caused by MRSA was reduced by the drugs in either their free forms or nanoparticulate forms; however, this reduction was not very significant. Neutrophils expressing Ly6G were clearly detected in the kidneys after MRSA infection. The number of neutrophils was markedly reduced after nanocarrier injection, indicating an efficient alleviation of infection-associated inflammation.

\section{Discussion}

Early initiation of the inhibition of bacterial growth and inflammation is crucial for the treatment of bacteremia and sepsis. The current therapy for mitigating bacteremia-induced sepsis is unsatisfactory for achieving a successful outcome. Improving drug delivery systems is one of the strategies for enhancing therapeutic efficiency against bacteremia. In our study, we carried out thorough analyses to assess the capability of treating bacteremia with NLCs incorporating both antibacterial and antiinflammatory drugs. We found that NLCs were more effective in eradicating MRSA than free drugs (Fig. 1). A similar trend was observed for inhibiting superoxide anion production by activated neutrophils (Fig. 2b). Our results demonstrate a large accumulation of intravenously injected NLCs in the kidneys (Fig. 3). This accumulation was beneficial for alleviating the renal dysfunction caused by bacteremia. Intravenous delivery of dual drug-loaded nanocarriers prevented the worsening of sepsis in mice and elevated the survival rate (Fig. 4a). Nanomedicine has been widely used due to its success in eradicating drug-resistant bacteria in vitro or in vivo [27-32]. We focused on the effect of nanocarriers encapsulating two drugs on the treatment of the bacteremia induced by MRSA. We also found that retinol incorporation in NLCs was beneficial for renal targeting in order to treat kidney injury.

The application of high-pressure homogenization and sonication was used to obtain NLCs of small particle size (Table 1). Particle diameters of $10-200 \mathrm{~nm}$ are most relevant for exerting biological effects [33]. For lipid-based 


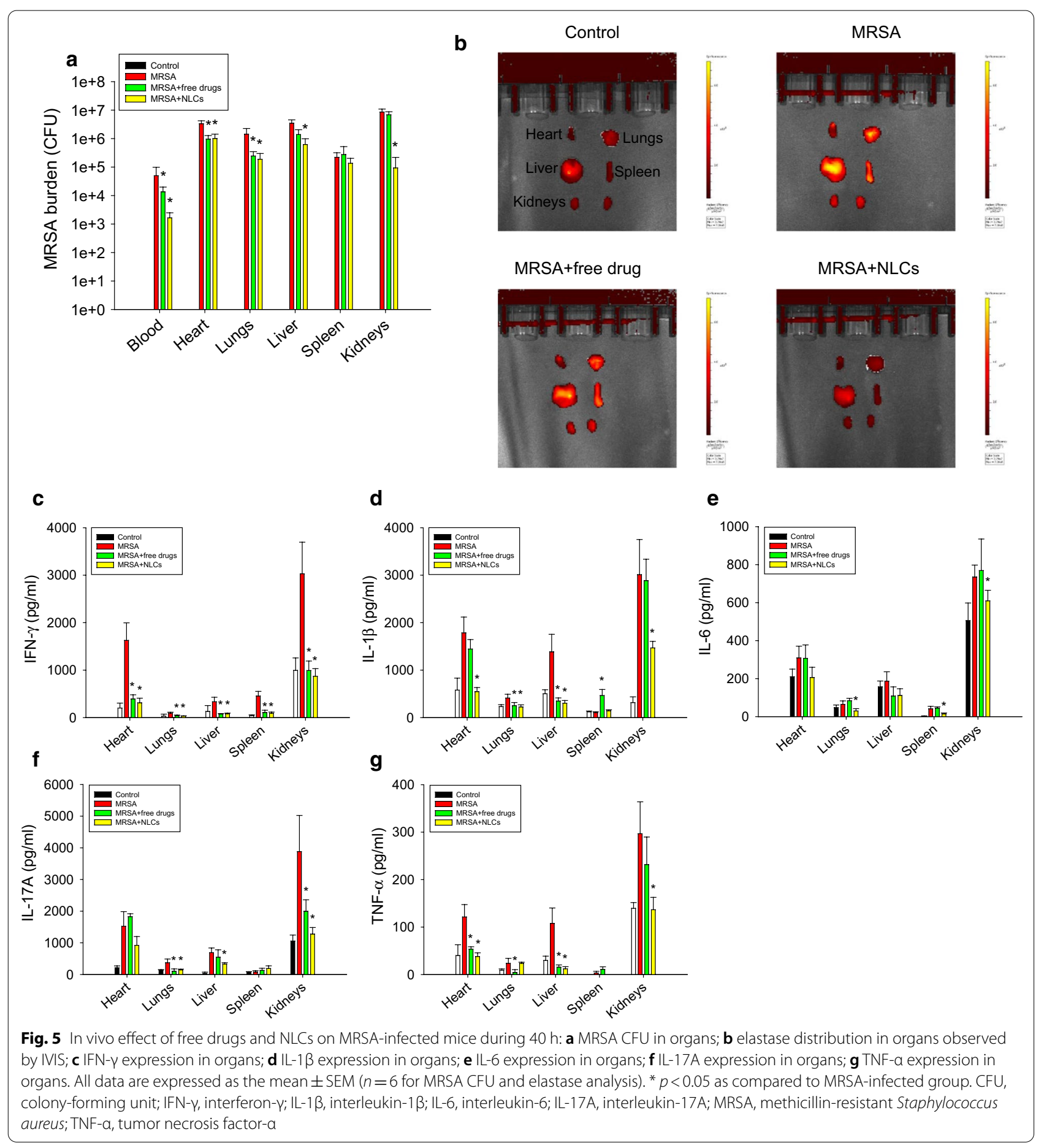

nanoparticles, a PDI of $\leq 0.3$ is considered to be acceptable [34]. Our nanosystems showed a PDI of 0.42 , and they can be considered highly polydisperse [35]. Nevertheless, the particle diameter was maintained at low size (171 $\mathrm{nm})$ with a similar PDI in the three batches $(0.42 \pm 0.04)$. We therefore concluded that the quality control of the nanocarriers was adequate. The nanocarriers prepared in the present study fit this criterion. Cationic nanoparticles are associated with hemolysis after intravenous administration [36]; therefore, we designed nanoparticles with a negative surface charge to prevent hemolysis. We incorporated deoxycholic acid into the nanoformulations 


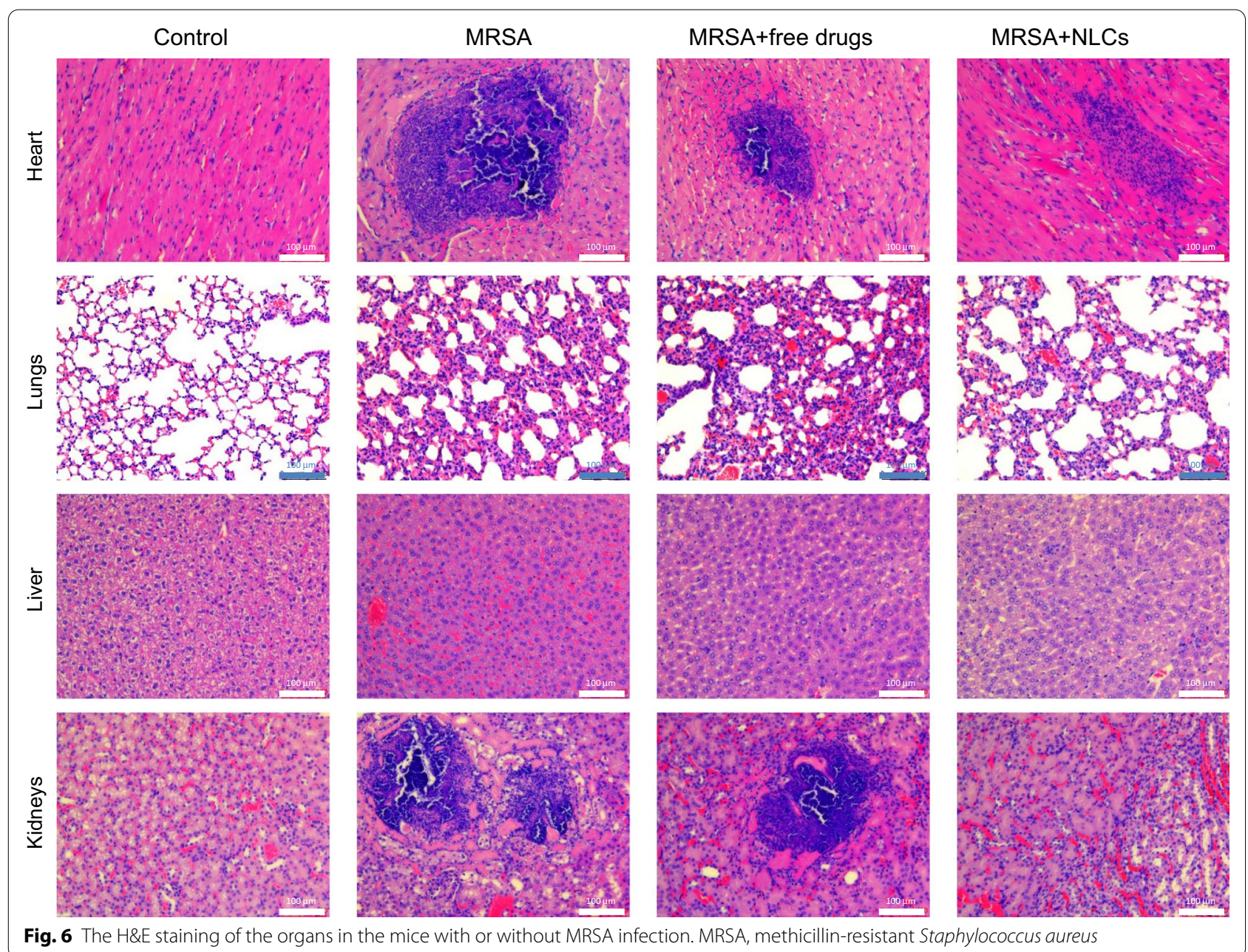

to obtain a negative charge of $-39 \mathrm{mV}$. This value was advantageous in showing sufficient storage stability because a zeta potential of $>30$ or $<-30 \mathrm{mV}$ indicates good electrostatic stabilization [37]. The high rate of rolipram encapsulation in the NLCs was attributed to the high lipophilicity of this compound, which allowed it to be retained in the lipid cores of the NLCs. Although ciprofloxacin is less lipophilic than rolipram, the encapsulation percentage of this drug reached nearly $90 \%$. The mixture of lipids in solid and liquid forms in the cores of NLCs leads to imperfection in the matrix, which allows greater space for loading both lipophilic and hydrophilic chemicals [38].

Ciprofloxacin, but not rolipram, demonstrated antiMRSA activity. The degree of MRSA suppression by ciprofloxacin was significantly reduced after combined treatment with rolipram in either the free or nanoparticulate forms (Table 2). A previous study [39] verified the possibility that ciprofloxacin could exhibit reduced antibacterial activity in the presence of PDE4 inhibitors.
In addition to their anti-inflammatory properties, PDE inhibitors have other bioactivities, such as antioxidative activity and protective activity against oxidative stress. Given the antibacterial mechanisms of ciprofloxacin, namely, the induction of reactive oxygen species to kill microbes, PDE inhibitors may restrict the antibacterial potency of ciprofloxacin. A possible drug-drug interaction between PDE4 inhibitors and ciprofloxacin was also observed in a previous investigation [40]. The combined treatment also exhibited a similar trend in its anti-inflammatory activity against activated neutrophils (Fig. 2b). The production of oxidative stress by ciprofloxacin may be the reason for the reduced ability of rolipram to inhibit superoxide anion production. The inclusion of ciprofloxacin in the nanoparticles inhibited MRSA growth compared to the free drugs (Fig. 1). Sustained and slow release of the drug from nanocarriers maintains drug retention near bacteria over a long period [9]. The surface of NLCs containing phosphatidylcholine and poloxamer 188 can easily fuse with the 

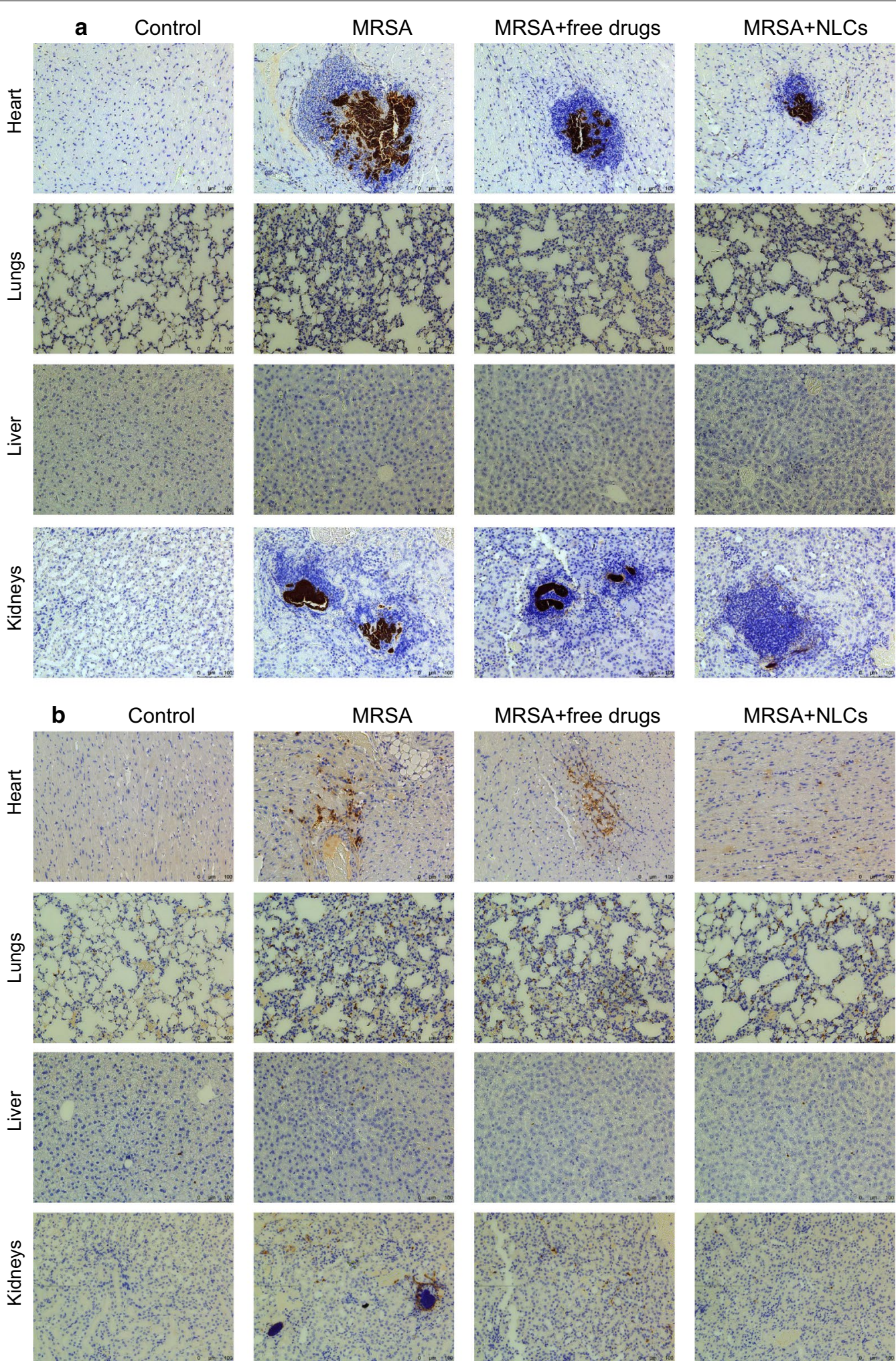

Fig. 7 The histology of the organs in the mice with or without MRSA infection: a MRSA distribution determined by S. aureus Rosenbach antibody; and $\mathbf{b}$ neutrophil distribution determined by recombinant anti-Ly6G antibody. MRSA, methicillin-resistant Staphylococcus aureus 
bacterial cell wall [41], resulting in the accumulation of antibacterial agents in large amounts in microbes.

Neutrophils are the major phagocytic cells that act as first-line responders in the fight against pathogenic microbes. Once uncontrolled activation of neutrophils occurs at the infected site, organs are possibly damaged. Neutrophils are recognized as essential players in acute inflammation, such as the inflammation elicited by bacteremia, sepsis, and traumatic hemorrhage [42]. Neutrophil stimulation is affected by the intracellular cAMP level, which can be regulated by PDE4 inhibition. Rolipram is a selective PDE4 inhibitor that reduces the number and activation of infiltrating neutrophils in inflammatory regions [43]. For intracellular drug delivery to inhibit neutrophil activation, nanocarriers must be internalized by neutrophils, and then, the drug is released. Our results demonstrated the easy ingestion of NLCs by neutrophils (Fig. 2c). A high zeta potential level, either positive or negative, has been established as a vital factor for assisting phagocytosis by neutrophils [44]. Soft nanoparticles can be deformed by cells during phagocytosis. This uptake is energetically less favorable [45]. Lipidbased nanoparticles can be classified as soft but not rigid structures that facilitate easy internalization by cells [46]. Neutrophil influx into peripheral organs mediates sepsis through superoxide generation. This oxidative stress induced by stimulated neutrophils is inactivated by the internalization of rolipram-loaded NLCs by neutrophils, which results in greater superoxide inhibition than drugs in free form (Fig. 2b).

An important feature for the treatment of bacteremia and sepsis is the appropriate penetration of drugs into tissues to limit organ damage. The therapeutic outcome can be improved when the drugs are delivered to the target organs. The biodistribution of intravenously injected NLCs showed the easy delivery of the nanoparticles to the kidneys (Fig. 3). An earlier study [12] also suggested higher deposition of NLCs in the kidneys than in other organs. Our NLCs possessed a size (150-180 nm) similar to that of the NLCs that were previously developed. The biodistribution results revealed a higher accumulation of NLCs with a higher concentration of retinol in the kidneys (Fig. 3). In the bloodstream, retinol is bound to specific retinol-binding proteins (RBPs), especially RBP4. RBPs play a principal role in promoting retinol transport in the body [47]. RBP4 is a plasma protein mainly secreted from the liver to transport retinol to peripheral organs via the bloodstream. The kidneys possess abundant RBP receptors with high retinol-binding activity [48]. In rats, approximately $50 \%$ of plasma retinol turnover is associated with the kidneys [49]. This may be the reason for the increased kidney deposition of the NLCs with high levels of incorporated retinol. The level of
RBP4 also increases during renal dysfunction but not during hepatic dysfunction [50]. Although the liver is also a main target of retinol transport, we observed minimal NLC deposition in this organ (Fig. 3). This result is quite different from the lipid-based nanoparticles prepared in our previous study [51], which demonstrated a large accumulation of retinol-loaded nanoparticles in the liver. The encapsulation percentage of retinol in the nanoparticles in the previous study was $0.02 \%$, which was much lower than that in the present study $(0.25 \%)$. It is difficult to compare the different nanocarriers since each nanosystem has its own intrinsic nature. In addition to the incorporation of materials, other factors, such as size, surface charge, and loaded drugs, also influence nanoparticle biodistribution. The biodistribution is nanocarrier-dependent and cannot be applied universally to all nanoformulations.

Once nanoparticles are administered into the blood, plasma proteins adsorb onto the particulate surface for opsonization. Opsonized nanoparticles are recognized by the mononuclear phagocyte system to be removed from circulation into the liver and spleen [52]. Nanoparticles with larger lipophilic surfaces tend to adsorb more proteins in circulation. The enhanced hydrophilic nature of the NLC surface can prevent uptake by the liver and spleen and thus prolong the circulation time [53]. The attachment of hydrophilic polymers, including polyethylene glycol, poloxamer, and chitosan, is effective for avoiding hepatic clearance [54]. Cationic nanoparticles show a high affinity for plasma proteins and parenchymal cells, leading to a broad distribution in the liver [55]. In our NLCs, the presence of poloxamers and a negative charge on their surfaces contributed to their low accumulation in the liver and spleen (Fig. 3). The nanoparticulate diameter is a key parameter for controlling their biodistribution. Nanocarriers larger than $200 \mathrm{~nm}$ are rapidly delivered to the liver and spleen because of complement activation [56, 57]. A previous study [58] also suggested that nanoparticles with sizes of $50-100 \mathrm{~nm}$ and $<50 \mathrm{~nm}$ are largely distributed in the hepatic parenchyma and spleen, respectively. According to this evidence, the size of our NLCs might have enabled their escape to enter the liver and spleen. The kidneys have fenestrated capillaries that facilitate the entrance of nanoparticles [55]; however, nanoparticles with a size $>6 \mathrm{~nm}$ cannot be removed from the kidneys [54]. Dual drug-loaded NLCs could penetrate but could not flow out of the kidneys, causing their accumulation in renal tissues. The uptake of NLCs by the brain is negligible, which could be due to the incorporation of poloxamer 188 to generate hydrophilic and steric surfaces. These surfaces are not conducive to delivery to the brain [12]. Limited brain targeting is favorable for rolipram delivery because this compound causes 
neurological disorders as side effects when it enters the brain.

In severe sepsis, the development of acute cardiac and renal dysfunction is commonly observed. S. aureus bacteremia has emerged as the most common cause of infective endocarditis [59]. Our results indicated decreased MRSA burden and elastase distribution in the heart after treatment with the combination of ciprofloxacin and rolipram (Fig. 5a and b). Moreover, NLCs exerted superior effects compared with free drugs. A previous study [60] demonstrated that ciprofloxacin is largely distributed in the cardiac cavity after injection. This observation explains the effectiveness of free ciprofloxacin in eradicating MRSA in the heart. Acute kidney injury is a major complication that arises due to sepsis. NLCs were helpful in inhibiting MRSA growth and elastase upregulation in the kidneys (Fig. 5a and b). This effect was not observed in the group treated with the free drugs, although PDE4 inhibition has been proven to attenuate acute renal failure in sepsis and endotoxemia $[24,61]$.

NLCs showed high efficiency for encapsulating ciprofloxacin and rolipram. Drugs encapsulated in nanoparticles can be protected from enzymatic attack to facilitate delivery to renal tissue. Ex vivo bioimaging in this study confirmed the large distribution of NLCs in the kidneys (Fig. 3). NLCs noticeably decreased the bacterial load and neutrophil recruitment in organs other than the kidneys, although the nanoparticle biodistribution in these organs was low. A large number of bacteria and neutrophils are present in bacteremic blood. It is suggested that NLCs inhibit MRSA growth and inflammation in circulation in the early stage of infection, followed by limited bacterial and neutrophil transport to peripheral organs. Ciprofloxacin molecules are rapidly cleared from circulation with $20 \%-40 \%$ protein binding $[61,62]$. The adsorption of plasma proteins to the nanoparticulate surface is generally low to evade degradation. NLCs with a size of $<200$ $\mathrm{nm}$, a poloxamer 188 -incorporated surface and negative zeta potential can be retained in the bloodstream for an extended period of time [63]. Poloxamer 188 increases the hydrophilicity of nanoparticulate surfaces, resulting in diminished macrophage recognition and phagocytosis. The crystalline solid lipids present in NLCs cause slower degradation of the lipid matrix to produce long-term circulation formulations [12]. Nanoparticle uptake by neutrophils might occur in circulation to arrest neutrophil activation and subsequent migration to organs.

The mortality associated with sepsis depends on the dysregulated host cytokine storm. Cytokines are inflammatory mediators that trigger inflammatory pathology and subsequent organ damage. The cytokines IL-1 $\beta$, IL- 6 , and TNF- $\alpha$ mediate the immunopathological features of sepsis and are released by neutrophils to exacerbate acute inflammation [64]. IL-6 is an early biomarker in bacteremia that is used to evaluate the inflammatory response $[6,65]$. TNF- $\alpha$ is produced in response to acute sepsis. IL- $1 \beta$ and IL- 6 enhance TNF- $\alpha$ expression to stimulate neutrophil recruitment. Activated neutrophils are the major inflammatory cells that express IL-17A, which can be used as a predictor of S. aureus bacteremia [66]. The organs of MRSA-infected rats showed increased levels of these cytokines (Fig. $5 \mathrm{c}$ to g). Our nanocarriers inhibited cytokine expression, suggesting their capacity to inhibit inflammation. The NLCs containing antibiotics and PDE4 inhibitors could inhibit MRSA growth and neutrophil activation to limit the cytokine storm caused by the bacteremia-induced inflammatory response. The acute inflammation induced by dysregulated cytokines during sepsis leads to vasodilation and apoptosis in different tissues [23]. After MRSA challenge, we observed congestion and necrosis in the heart, lungs, and kidneys (Fig. 6). The increased permeability of blood vessels allowed neutrophils to migrate from the blood to peripheral organs. Histological analysis of Ly6G showed neutrophil accumulation in the organs (Fig. 7b), while NLCs inhibited the entrance of neutrophils into organs and thus prevented organ disruption. The organ failure caused by the host response to infection is a predominant mechanism of sepsis-related death. The mortality associated with bacteremia is dependent on cytokine overexpression, and attenuated cytokine release is a prerequisite for reducing mortality [61]. In this study, the mortality rate notably increased in the MRSA-infected mice (Fig. 4a), suggesting successful creation of the bacteremia model. Rolipram was found to be useful in improving cardiac and renal function during sepsis, thus promoting the survival of the animals [24]. Our data confirmed the role of rolipram in improving survival. This amelioration was further enhanced by the encapsulation of rolipram in lipid-based nanocarriers. The NLCs suppressed the release of proinflammatory cytokines in bacteremic mice, decreased the MRSA load, and increased survival.

There were some limitations in our study. The bacteremia model established in this study and its clinical relevance are still unclear. Treatment with drugs prior to the induction of bacteremia led to an effective and stable plasma drug concentration. However, in clinical practice, it is difficult to administer drugs before bacteremia onset; thus, our method of using NLCs to treat bacteremia could be used for prevention but not for therapy with rigorous definitions. The experimental periods for the in vivo study ( $40 \mathrm{~h}$ and 4 days) were too short to offer more information regarding the long-term outcome and augment the importance of our work. Additional basic studies are required to verify any potential effect of NLCs on bacteremia. The combination of free ciprofloxacin 
and rolipram might cause interactions that would lower their antimicrobial and anti-inflammatory activities, but the detailed mechanisms are not completely understood. Further investigation is required to explore the inherent mechanisms.

\section{Conclusions}

The present work demonstrated the successful preparation of dual drug-loaded NLCs to enhance the efficiency of bacteremia treatment. The encapsulation of ciprofloxacin and rolipram into lipid-based nanoparticles was nearly complete. In vitro evaluation revealed superior antibacterial and anti-inflammatory potencies of the NLCs compared to the combination of free drugs using MRSA and neutrophils as models, respectively. Intravenously injected NLCs were deposited primarily in the kidneys due to the incorporation of retinol in the nanoparticles. Treatment with NLCs encapsulating both ciprofloxacin and rolipram improved bacterial clearance, inhibited elastase expression, and prevented organ damage in rats infected with MRSA. The nanocarriers were found to suppress the cytokine overexpression in peripheral organs and improve the survival rate of rats with bacteremia-induced sepsis. The intravenous nanocarriers possibly mitigated MRSA growth and suppressed neutrophil activation in circulation, followed by minimizing MRSA and neutrophil transport to organs. The combination of anti-MRSA and anti-inflammatory nanocarriers relieved the acute inflammation caused by MRSA infection and thus showed potential application for bacteremia treatment.

\section{Methods}

\section{Preparation of NLCs}

The NLCs were fabricated by the homogenization-sonication method [41]. The aqueous and lipid phases were prepared separately. Three hundred fifty milligrams of Poloxamer 188 and $100 \mathrm{mg}$ of deoxycholic acid were dissolved in water $(8.89 \mathrm{ml})$. The lipid phase consisted of squalene $(400 \mathrm{mg})$, hexadecyl palmitate $(100 \mathrm{mg})$, soybean phosphatidylcholine (Phospholipon $80 \mathrm{H}, 150 \mathrm{mg}$ ), retinol $(25 \mathrm{mg})$, ciprofloxacin $(5 \mathrm{mg})$, and rolipram $(2 \mathrm{mg})$. Both phases were heated at $85^{\circ} \mathrm{C}$ for $15 \mathrm{~min}$. Then, the aqueous phase was dispersed into the lipid phase with high-shear homogenization at $12,000 \mathrm{rpm}$ for $20 \mathrm{~min}$. The mixture was further sonicated using a probetype sonicator at $35 \mathrm{~W}$ for $15 \mathrm{~min}$. The final weight of the NLCs was $10 \mathrm{~g}$.

\section{Estimation of the particle diameter and zeta potential}

The average diameter and zeta potential of the NLCs were evaluated by a Nano ZS90 analyzer (Malvern). All the samples were diluted 100-fold with water. Each sample was analyzed in triplicate.

\section{The encapsulation efficiency of the drugs}

The encapsulation percentage of ciprofloxacin and rolipram was calculated by using the ultracentrifugation method to separate the encapsulated drugs from the free forms. The NLCs were centrifuged at $48,000 \mathrm{x} g$ and $4^{\circ} \mathrm{C}$ for $40 \mathrm{~min}$. The free drugs in the supernatant and encapsulated drugs in the precipitate were analyzed by highperformance liquid chromatography (HPLC), whose setups for ciprofloxacin and rolipram have been previously described $[67,68]$.

\section{Drug release from the nanoparticles}

The drug release of the ciprofloxacin- and rolipramloaded NLCs was studied by the dialysis bag diffusion method. The drug-loaded control vehicle or NLCs (5 $\mathrm{ml}$ ) were dispersed in a dialysis bag, and the dialysis bag (Cellu-Sep T2 with an MW cutoff of 6000-8000 Da) was then incubated in a beaker containing $100 \mathrm{ml}$ of $\mathrm{pH} 7.4$ phosphate buffer. The free control was drugs dissolved in $35 \%$ ethanol/pH 7.4 buffer. The beaker was placed on a magnetic stirrer with a stirring rate of $100 \mathrm{rpm}$, and the temperature of the assembly was maintained at $37^{\circ} \mathrm{C}$. Samples $(1 \mathrm{ml})$ were collected at definite time intervals and replaced with equal amounts of fresh buffer. After suitable dilutions, the samples were analyzed using HPLC.

\section{Minimum bactericidal concentration (MBC)}

MRSA (ATCC33591) was purchased from the American Type Culture Collection. The MRSA suspension was diluted in tryptone soy broth to obtain a concentration of $\mathrm{OD}_{600}=0.01$. The TSB plate was incubated with different concentrations $(0-31.25 \mu \mathrm{g} / \mathrm{ml})$ of ciprofloxacin with or without rolipram either in their free or nanoparticulate forms at $37^{\circ} \mathrm{C}$ for $20 \mathrm{~h}$. The CFU was then counted. The $\mathrm{MBC}$ was defined as the lowest ciprofloxacin concentration required to kill $\geq 99.9 \%$ of MRSA (ATCC33591).

\section{Time-response MRSA growth inhibition}

The inhibition of MRSA growth by the drugs in their free or nanoparticulate form within $24 \mathrm{~h}$ was measured in 96-well plates. Ciprofloxacin $(0.5$ or $1 \mu \mathrm{g} / \mathrm{ml})$, rolipram $(0.2$ or $0.4 \mu \mathrm{g} / \mathrm{ml})$, or their combination was incubated with MRSA $\left(\mathrm{OD}_{600}=0.01\right)$ at $35^{\circ} \mathrm{C}$ for $24 \mathrm{~h}$. The absorbance of each well was measured at $600 \mathrm{~nm}$ to detect MRSA growth in real-time.

\section{Isolation of human neutrophils}

Neutrophils from healthy donors between the ages of $20-30$ years old were isolated by using a protocol 
approved by the Institutional Review Board at Chang Gung Memorial Hospital. Written informed consent was obtained from every subject. The neutrophils were purified by sedimentation prior to centrifugation in a Ficoll Hypaque gradient and the hypotonic lysis of erythrocytes [69].

\section{Neutrophil viability}

Lactate dehydrogenase (LDH) is an indicator of cellular membrane leakage and cell viability. The commercial kit CytoTox 96 (Promega) was used to estimate the LDH levels. The detailed protocol was previously described [70].

\section{Superoxide anion production by neutrophils}

Superoxide anion release from primary neutrophils was assessed using Ferricytochrome $c$ [71]. Briefly, neutrophils $\left(6 \times 10^{5}\right.$ cells $\left./ \mathrm{ml}\right)$ were incubated with ferricytochrome $c(0.5 \mathrm{mg} / \mathrm{ml})$ and $\mathrm{CaCl}_{2}(1 \mathrm{mM})$ at $37^{\circ} \mathrm{C}$. Neutrophils were treated with ciprofloxacin $(0.75-75$ $\mathrm{nM})$, rolipram $(0.3-30 \mathrm{nM})$, or their combination in free or nanoparticulate form for $10 \mathrm{~min}$. Then, the neutrophils were activated by formyl-methionyl-leucyl phenylalanine (fMLF, $0.1 \mu \mathrm{M})$ and cytochalasin B $(1 \mu \mathrm{g} /$ $\mathrm{ml}$ ). Ferricytochrome $c$ reduction was monitored by measuring the absorbance at $550 \mathrm{~nm}$.

\section{Nanoparticle uptake by neutrophils}

NLCs were labeled with $0.1 \mathrm{mg} / \mathrm{ml}$ rhodamine 800 dye to observe their ingestion by neutrophils. Neutrophils $\left(1 \times 10^{7}\right.$ cells $\left./ \mathrm{ml}\right)$ were incubated with NLCs $(5$ or 10 $\mu \mathrm{l})$ at $37^{\circ} \mathrm{C}$ for $5 \mathrm{~min}$, and the reaction was stopped by adding Hank's balanced salt solution at $4^{\circ} \mathrm{C}$. The neutrophils were imaged using confocal microscopy. The fluorescence intensity of the rhodamine-labeled NLCs was also quantified by flow cytometry.

\section{Animals}

Male Sprague-Dawley rats (200-300 g) and Balb/c mice (20-25 g) were purchased from Lasco Biotechnology (Taipei, Taiwan). All the animals were treated in accordance with protocols approved by the Institute of Animal Care and the Use Committee of Chang Gung University.

\section{Biodistribution of NLCs in rats}

Near-infrared (NIR) dye (iFluor 790; 0.08\%) was incorporated into the NLCs to monitor the biodistribution of intravenously injected NLCs. The rats were anesthetized with Zoletil $50(30 \mathrm{mg} / \mathrm{kg})$ and xylazine $(6 \mathrm{mg} / \mathrm{kg})$. The NLCs $(0.8 \mathrm{ml} / \mathrm{kg})$ were administered via the tail vein.
The rats were sacrificed after $2 \mathrm{~h}$. The organs were harvested to detect the NIR signals with an in vivo imaging system (IVIS, Pearl Impulse Imaging System, Li-Cor). The NIR signal intensity was quantified by Pearl Impulse software.

\section{Bacteremia model induced by MRSA in mice}

A mouse model of bacteremia was induced according to a previous study with some modifications [72]. The mice were randomly assigned into four groups: (i) uninfected control receiving PBS, (ii) MRSA infection, (iii) MRSA infection with intravenous injection of two drugs in $10 \%$ ethanol, and (iv) MRSA infection with intravenous injection of dual drug-loaded NLCs. Bacteremia was induced by intravenous injection of MRSA $\left(1 \times 10^{7} \mathrm{CFU}\right)$. The mice were administered NLCs or $10 \%$ ethanol containing ciprofloxacin $(2.5 \mathrm{mg} / \mathrm{kg})$ and rolipram $(1 \mathrm{mg} / \mathrm{kg})$ $24 \mathrm{~h}$ before injection with MRSA. Mortality was monitored and recorded over four days with a camera. The mice were sacrificed at $40 \mathrm{~h}$ and $4 \mathrm{~d}$ postinfection to estimate the MRSA CFU counts in the blood and peripheral organs.

\section{Elastase in organs}

The neutrophil elastase 680 FAST imaging agent (PerkinElmer) was used to observe the levels of neutrophil elastase in vivo. Four hours after the intravenous injection of the imaging agent $(100 \mu \mathrm{l})$, the animals were sacrificed to harvest the organs. The harvested organs were observed and photographed using a fluorescence IVIS system (Lumina LT Series III, PerkinElmer).

\section{Cytokine expression in organs}

Samples from the organs of mice were used to measure the levels of cytokines, including IFN- $\gamma$, IL-1 $\beta$, IL-6, IL$17 \mathrm{~A}$, and TNF- $\alpha$, using commercial kits (BioLegend), following the manufacturer's instructions.

\section{Histological observation}

Specimens from different organs were immersed in $10 \%$ formaldehyde, embedded in paraffin, and then sliced into 5- $\mu \mathrm{m}$-thick sections for $\mathrm{H} \& \mathrm{E}$ staining. We also examined MRSA and Ly6G in the organs by immunohistochemistry. The organ sections were incubated with the relevant antibodies ( $S$. aureus Rosenbach antibody for MRSA and recombinant anti-Ly6G antibody for neutrophils) for $1 \mathrm{~h}$ and then incubated with biotinylated donkey anti-rabbit IgG for $20 \mathrm{~min}$. The sections were observed by optical microscopy. 


\section{Statistical analysis}

The data shown in this work are presented the mean and the standard error of the mean. Significant differences between different groups were examined by the Kruskal-Wallis method. Individual differences were evaluated post hoc by Dunn's test. Significance was demonstrated by $p<0.05$.

\section{Supplementary Information}

The online version contains supplementary material available at https://doi. org/10.1186/s12951-021-00789-5.

Additional file 1: Figure S1. The release kinetics of ciprofloxacin androlipram from free control and NLCs.

\section{Acknowledgements}

The authors are grateful for the financial support from Ministry of Science and Technology of Taiwan (MOST-107-2320-B-182-016-MY3) Chang Gung Memorial Hospital (CMRPG3H0671-3)

\section{Authors' contributions}

$C C L, F C L$, and JYF designed the research. CCL, HPY, SCY and YSD performed the experiments and collected and analyzed the data. CCL, HPY, and AA validated the results. HPY, SCY, and AA wrote the manuscript text and prepared the figures. FCL and JYF have read critically and edited the manuscript. CCL and JYF acquired the funding. All authors reviewed the manuscript. All authors read and approved the final manuscript.

\section{Availability of data and materials}

The datasets used and/or analysed during the current study are available from the corresponding author on reasonable request.

\section{Ethics approval and consent to participate}

Not applicable.

\section{Consent for publication}

Not applicable.

\section{Competing interests}

The authors declare that they have no competing interests.

\begin{abstract}
Author details
${ }^{1}$ Department of Anesthesiology, Chang Gung Memorial Hospital, 5 Fuxing Street, Kweishan, Taoyuan 333, Taiwan. ${ }^{2}$ School of Medicine, College of Medicine, Chang Gung University, Kweishan, Taoyuan, Taiwan. ${ }^{3}$ Department of Cosmetic Science, Providence University, Taichung, Taiwan. ${ }^{4}$ Department of Pharmaceutics, College of Pharmacy, Prince Sattam Bin Abdulaziz University, Al Kharj, Saudi Arabia. ${ }^{5}$ Pharmaceutics Laboratory, Graduate Institute of Natural Products, Chang Gung University, 259 Wen-Hwa 1st Road, Kweishan, Taoyuan 333, Taiwan. ${ }^{6}$ Research Center for Food and Cosmetic Safety and Research Center for Chinese Herbal Medicine, Chang Gung University of Science and Technology, Kweishan, Taoyuan, Taiwan.
\end{abstract}

Received: 6 November 2020 Accepted: 2 February 2021

Published online: 15 February 2021

\section{References}

1. Bergin SP, Holland TL, Flowler VG Jr, Tong SYC. Bacteremia, sepsis, and infective endocarditis associated with Staphylococcus aureus. Curr Top Microbiol Immunol. 2017;409:263-96.

2. Holland TL, Arnold C, Flowler Jr. VG. Clinical management of Staphylococcus aureus bacteremia. A review. JAMA. 2014;312:1330-41.

3. Corl KA, Zeba F, Caffrey AR, Hermenau M, Lopes V, Phillips G, Merchant RC, Levy MM, LaPlante KL. Delay in antibiotic administration is associated with mortality among septic shock patients with Staphylococcus aureus bacteremia. Crit Care Med. 2020;48:525-32.

4. Minejima E, Bensman J, She RC, Mack WJ, Tuan Tran M, Ny P, Lou M, Yamaki J, Nieberg P, Ho J, Wong-Beringer A. A dysregulated balance of proinflammatory and anti-inflammatory host cytokine response early during therapy predicts persistence and mortality in Staphylococcus aureus bacteremia. Crit Care Med. 2016;44:671-9.

5. Annane D, Aegerter P, Jars-Guincestre MC, Guidet B. Current epidemiology of septic shock: the CUB-Rea network. Am J Respir Crit Care Med. 2003; 168:165-72

6. Salas DE, Minejima E, Wu J, Fang C, Wang J, She R, Nieberg P, WongBeringer A. Staphylococcus aureus bacteremia in patients not meeting sepsis criteria: clinical features, host immune response and outcomes. J Clin Med Ther. 2017:2:27.

7. Rello J, Valenzuela-Sánchez F, Ruis-Rodriguez M, Moyano S. Sepsis: a review of advances in management. Adv Ther. 2017:34:2393-411.

8. Hassoun A, Linder PK, Friedman B. Incidence, prevalence, and management of MRSA bacteremia across patient populations-a review of recent developments in MRSA management and treatment. Crit Care. 2017;21:211.

9. Aljuffali IA, Huang CH, Fang JY. Nanomedical strategies for targeting skin microbiomes. Curr Drug Metab. 2015;16:255-71.

10. Ikoba U, Peng H, Li H, Miller C, Yu C, Wang Q. Nanocarriers in therapy of infections and inflammatory diseases. Nanoscale. 2015;7:4291.

11. Yeh YC, Huang TH, Yang SC, Chen CC, Fang JY. Nano-based drug delivery or targeting to eradicate bacteria for infection mitigation: a review of recent advances. Front Chem. 2020;8:286.

12. Beloque A, Solinís MA, Delgado A, Évora C, del Pozo-Rodríguez A, Rodríguez-Gascón A. Biodistribution of nanostructured lipid carriers (NLCs) after intravenous administration to rats: influence of technological factors. Eur J Pharm Biopharm. 2013:84:309-14.

13. Hattori Y, Hattori K, Suzuki T, Matsuda N. Recent advances in the pathophysiology and molecular basis of sepsis-associated organ dysfunction: novel therapeutic implications and challenges. Pharmacol Ther. 2017; 177:56-66.

14. Liappis AP, Kan VL, Rochester CG, Simon GL. The effect of statins on mortality in patients with bacteremia. Clin Infect Dis. 2001;33:1352-7.

15. Sung $\mathrm{PH}$, Chiang $\mathrm{HJ}$, Chen $\mathrm{CH}$, Chen $\mathrm{YL}$, Huang $\mathrm{TH}$, Zhen $\mathrm{YY}$, Chang MW, Liu CF, Chung SY, Chen YL, Chai HT, Sun CK, Yip HK. Combined therapy with adipose-derived mesenchymal stem cells and ciprofloxacin against acute urogenital organ damage in rat sepsis syndrome induced by intrapelvic injection of cecal bacteria. Stem Cells Transl Med. 2016;5:782-92.

16. Choo EJ, Chambers HF. Treatment of methicillin-resistant Staphylococcus aureus bacteremia. Infect Chemother. 2016;48:267-73.

17. Amodio-Groton M, Madu A, Madu CN, Briceland LL, Seligman M, McMaster $\mathrm{P}$, Miller $\mathrm{MH}$. Sequential parenteral and oral ciprofloxacin regimen versus parenteral therapy for bacteremia: a pharmacoeconomic analysis. Ann Pharmacother. 1996;30:596-602.

18. Hur J, Lee A, Hong J, Jo WY, Cho OH, Kim S, Bae IG. Staphylococcus aureus bacteremia originating from urinary tract infections: a case report and literature review. Infect Chemother. 2016;48:136-9.

19. Lo CL, Lee CC, Li CW, Lin MC, Hsueh PR, Lee NY, Ko WC. Fluoquinolone therapy for bloodstream infections caused by extended-spectrum betalactamase-producing Escherichia coli and Klebsiella pneumonia. J Microb Immunol Infect. 2017;50:355-61.

20. Li H, Zuo J, Tang W. Phosphodiesterase-4 inhibitors for the treatment of inflammatory diseases. Front Pharmacol. 2018;9:1048.

21. Chang X, Hu LF, Ma XJ, Yin J, Liu XY, Li JB. Influence of roflumilast on sepsis mice through the JAK/STAT signaling pathway. Eur Rev Med Pharmacol Sci. 2019;23:1335-41.

22. Dalamaga M, Karampela I, Mantzoros CS. Commentary: phosphodiesterase 4 inhibitors as potential adjunct treatment targeting the cytokine storm in COVID-19. Metab Clin Exp. 2020;109:154282.

23. Xu X, Liao L, Hu B, Jiang H, Tan M. Roflumilast, a phosphodiesterase-4 (PDE4) inhibitor, alleviates sepsis-induced acute kidney injury. Med Sci Monit. 2020;26:e921319.

24. Sims CR, Singh SP, Mu S, Gokden N, Zakaria D, Nguyen TC, Mayeux PR. Rolipram improves outcome in a rat model of infant sepsis-induced cardiorenal syndrome. Front Pharmacol. 2017;8:237. 
25. Lu X, Wang J, Chen X, Jiang Y, Pan ZK. Rolipram protects mice from Gramnegative bacterium Escherichia coli-induced inflammation and septic shock. Sci Rep. 2020;10:175.

26. Christaki E, Giamarellos-Bourboulis EJ. The complex pathogenesis of bacteremia. From antimicrobial clearance mechanisms to the genetic background of the host. Virulence. 2014;5:57-65.

27. Hindi KM, Ditto AJ, Panzner MJ, Medvetz DA, Han DS, Hovis CE, Hilliard JK, Taylor JB, Yun YH, Cannon CL, Youngs WJ. The antimicrobial efficacy of sustained release silver-carbene complex-loaded L-tyrosine polyphosphate nanoparticles: characterization, in vitro and in vivo studies. Biomaterials. 2009;30:3771-9.

28. Ansari MA, Khan HM, Khan AA, Pal R, Cameotra SS. Antibacterial potential of $\mathrm{Al}_{2} \mathrm{O}_{3}$ nanoparticles against multidrug resistance strains of Staphylococcus aureus isolated from skin exudates. J Nanopart Res. 2013;15:1970

29. Sun D, Zhang W, Li N, Zhao Z, Mou Z, Yang E, Wang W. Silver nanoparticles-quercetin conjugation to siRNA against drug-resistant Bacillus subtilis for effective gene silencing: in vitro and in vivo. Mater Sci Eng C. 2016;63:522-34

30. Gao F, Xu L, Yang B, Fan F, Yang L. Kill the real with the fake: eliminate intracellular Staphylococcus aureus using nanoparticle coated with its extracellular vesicle membrane as active-targeting drug carrier. ACS Infect Dis. 2019;5:218-27.

31. Zhang C, Zhao W, Bian C, Hou X, Deng B, McComb DW, Chen X, Dong Y. Antibiotic-derived lipid nanoparticles to treat intracellular Staphylococcus aureus. ACS Appl Bio Mater. 2019;2:1270-7.

32. Vanamala K, Tatiparti K, Bhise K, Sau S, Scheetz MH, Rybak MJ, Andes D, lyer AK. Novel approaches for the treatment of methicillin-resistant Staphylococcus aureus: using nanoparticles to overcome multidrug resistance. Drug Discov Today. 2020. https://doi.org/10.1016/j.drudis.2020.10.011.

33. Hickey JW, Santos JL, Williford JM, Mao HQ. Control of polymeric nanoparticle size to improve therapeutic delivery. J Control Release. 2015;219:536-47.

34. Zhang J, Froelich A, Michniak-Kohn B. Topical delivery of meloxicam using liposome and microemulsion formulation approaches. Pharmaceutics. 2020:12:282.

35. Bhattacharjee S. DLS and zeta potential — what they are and what they are not? J Control Release. 2016;235:337-51.

36. Dobrovolskaia MA, Aggarwal P, Hall JB, McNeil SE. Preclinical studies to understand nanoparticle interaction with the immune system and its potential effects on nanoparticle biodistribution. Mol Pharm. 2008:5:487-95.

37. Alcantara KP, Zulfakar MH, Castillo AL. Development, characterization and pharmacokinetics of mupirocin-loaded nanostructured lipid carriers (NLCs) for intravascular administration. Int J Pharm. 2019;571:118705.

38. Zhang K, Lv S, Li X, Feng Y, Li X, Liu L, Li S, Li Y. Preparation, characterization, and in vivo pharmacokinetics of nanostructured lipid carriers loaded with oleanolic acid and gentiopicrin. Int J Nanomed. 2013;8:3227-39.

39. Masadeh MM, Alzoubi KH, Khabour OF, Al-Azzam SI. Ciprofloxacininduced antibacterial activity is attenuated by phosphodiesterase inhibitors. Curr Ther Res. 2014;77:14-7.

40. Vignola AM. PDE4 inhibitors in COPD—a more selective approach to treatment. Respir Med. 2004;98:495-503.

41. Alalaiwe A, Wang PW, Lu PL, Chen YP, Fang JY, Yang SC. Synergistic antiMRSA activity of cationic nanostructured lipid carriers in combination with oxacillin for cutaneous application. Front Microbiol. 2018;9:1493.

42. Lin MH, Lin CF, Yang SC, Hung CF, Fang JY. The interplay between nanoparticles and neutrophils. J Biomed Nanotechnol. 2018;14:66-85.

43. Tavares LP, Garcia CC, Vago JP, Queiroz-Junior CM, Galvão I, David BA, Rachid MA, Silva PMR, Russo RC, Teixeira MM, Sousa LP. Inhibition of phosphodiesterase-4 during pneumococcal pneumonia reduces inflammation and lung injury in mice. Am J Respir Cell Mol Biol. 2016;55:24-34

44. Kelley WJ, Fromen CA, Lopez-Cazares G, Eniola-Adefeso O. PEGylation of model drug carriers enhances phagocytosis by primary human neutrophils. Acta Biomater. 2018;79:283-93.

45. Boraschi D, Italiani P, Palomba R, Decuzzi P, Duschl A, Fadeel B, Moghimi SM. Nanoparticles and innate immunity: new perspectives on host defense. Semin Immunol. 2017;34:33-51.

46. Yu HP, Liu FC, Umoro A, Lin ZC, Elzoghby AO, Hwang TL, Fang JY. Oleic acid-based nanosystems for mitigating acute respiratory distress syndrome in mice through neutrophil suppression: how the particulate size affects therapeutic efficiency. J Nanobiotechnol. 2020;18:25.
47. O'Byrne SM, Blaner WS. Retinol and retinyl esters: biochemistry and physiology. J Lipid Res. 2013;54:1731-43.

48. Smeland S, Bjerknes T, Malaba L, Eskild W, Norum KR, Blomhoff R. Tissue distribution of the receptor for plasma retinol-binding protein. Biochem $J$. 1995:305:419-24

49. Raila J, Willnow TE, Schweigert FJ. Megalin-mediated reuptake of retinol in the kidneys of mice is essential for vitamin A homeostasis. J Nutr. 2005;135:2512-6.

50. Frey SK, Nagl B, Henze A, Raila J, Schlosser B, Berg T, Tepel M, Zidek W, Weickert MO, Pfeiffer AFH, Schweigert FJ. Isoforms of retinol binding protein (RBP4) are increased in chronic diseases of the kidney but not of the liver. Lipids Health Dis. 2008;7:29.

51. Pan TL, Wang PW, Hung CF, Aljuffali IA, Dai YS, Fang JY. The impact of retinol loading and surface charge on the hepatic delivery of lipid nanoparticles. Colloids Surf B Biointerf. 2016;141:584-94.

52. Shah NB, Vercellotti GM, White JG, Fegan A, Wagner CR, Bischof JC. Bloodnanoparticle interactions and in vivo biodistribution: impact of surface PEG and ligand properties. Mol Pharm. 2012;9:2146-55.

53. Su Z, Niu J, Xiao Y, Ping Q, Sun M, Huang A, You W, Sang X, Yuan D. Effect of octreotide-polyethylene glycol(100) monostearate modification on the pharmacokinetics and cellular uptake of nanostructured lipid carrier loaded with hydroxycamptothecine. Mol Pharm. 2011;8:1641-51.

54. Zhao Z, Ukidve A, Krishnan V, Mitragotri S. Effect of physicochemical and surface properties on in vivo fate of drug nanocarriers. Adv Drug Deliv Rev. 2019;143:3-21.

55. Javidi J, Haeri A, Nowroozi F, Dadashzadeh S. Pharmacokinetics, tissue distribution and excretion of $\mathrm{Ag}_{2} \mathrm{~S}$ quantum dots in mice and rats: the effects of injection dose, particle size and surface charge. Pharm Res. 2019;36:46.

56. Almeida JPM, Chen AL, Foster A, Drezek R. In vivo biodistribution of nanoparticles. Nanomedicine. 2011;6:815-35.

57. Hoshyar N, Gray S, Han H, Bao G. The effect of nanoparticle size on in vivo pharmacokinetics and cellular interaction. Nanomedicine. 2016;11:673-92.

58. Xue W, Liu Y, Zhang N, Yao Y, Ma P, Wen H, Huang S, Luo Y, Fan H. Effects of core size and PEG coating layer of iron oxide nanoparticles on the distribution and metabolism in mice. Int J Nanomed. 2018;13:5719-31.

59. Dayan GH, Mohamed N, Scully IL, Cooper D, Begier E, Eiden J, Jansen KU, Gurtman A, Anderson AS. Staphylococcus aureus: the current state of disease, pathophysiology and strategies for prevention. Expert Rev Vaccines. 2016;15:1373-92.

60. De Winter F, Van de Wiele C, Dumont F, Van Durme J, Solanki K, Britton K, Slegers G, Dierckx RA, Thierens H. Biodistribution and dosimetry of ${ }^{99 \mathrm{~m} T c-}$ ciprofloxacin, a promising agent for the diagnosis of bacterial infection. Eur J Nucl Med. 2001;28:570-4.

61. Tofovic SP, Zacharia LC, Carcillo JA, Jackson EK. Inhibition of cytokine release by and cardiac effects of type IV phosphodiesterase inhibition in early, profound endotoxaemia in vivo. Clin Exp Pharmacol Physiol. 2000:27:787-92.

62. Wise R, Lockley RM, Webberly M, Dent J. Pharmacokinetics of intravenously administered ciprofloxacin. Antimicrob Agents Chemother. 1984;26:208-10.

63. Luan J, Zhang D, Hao L, Qi L, Liu X, Guo H, Li C, Guo Y, Li T, Zhang Q, Zhai G. Preparation, characterization and pharmacokinetics of amoitone B-loaded long circulating nanostructured lipid carriers. Colloids Surf B Biointerf. 2014;114:255-60.

64. Qin M, Qiu Z. Changes in TNF- $a, \mathrm{IL}-6, \mathrm{IL}-10$ and VEGF in rats with ARDS and the effects of dexamethasone. Exp Ther Med. 2019:17:383-7.

65. Abe R, Oda S, Sadahiro T, Nakamura M, Hirayama Y, Tateishi Y, Shinozaki K, Hirasawa H. Gram-negative bacteremia induces greater magnitude of inflammatory response than Gram-positive bacteremia. Crit Care. 2010;14:27.

66. Cao Y, Guimaraes AO, Peck MC, Mayba O, Ruffin F, Hong K, CarrascoTriguero M, Fowler VG Jr, Maskarinec SA, Rosenberger CM. Risk stratification biomarkers for Staphylococcus aureus bacteremia. Clin Transl Immunol. 2020;9:e1110.

67. Fang $C L$, Wen CJ, Aljuffali IA, Sung CT, Huang CL, Fang JY. Passive targeting of phosphatiosomes increases rolipram delivery to the lungs for treatment of acute lung injury: an animal study. J Contro Release. 2015;213:69-78. 
68. Hsu CY, Sung CT, Aljuffali IA, Chen CH, Hu KY, Fang JY. Intravenous anti-MRSA phosphatiosomes mediate enhanced affinity to pulmonary surfactants for effective treatment of infectious pneumonia. NanomedNanotechnol Biol Med. 2018;14:215-25.

69. Yu HP, Hsieh PW, Chang YJ, Chung PJ, Kuo LM, Hwang TL. 2-(2-Fluorobenzamido)benzoate ethyl ester (EFB-1) inhibits superoxide production by human neutrophils and attenuates hemorrhagic shock-induced organ dysfunction in rats. Free Radic Biol Med. 2011;50:1737-48.

70. Liu FC, Yu HP, Lin CY, Elzoghby AO, Hwang TL, Fang JY. Use of cilomilastloaded phosphatiosomes to suppress neutrophilic inflammation for attenuating acute lung injury: the effect of nanovesicular surface charge. J Nanobiotechnol. 2018;16:35.

71. Yang SC, Chung PJ, Ho CM, Kuo CY, Hung MF, Huang YT, Chang WY, Chang YW, Chan KH, Hwang TL. Propofol inhibits superoxide production, elastase release, and chemotaxis in formyl peptide-activated human neutrophils by blocking formyl peptide receptor 1. J Immunol. 2013;190:6511-9.

72. Veloso TR, Chaouch A, Roger T, Giddey M, Vouillamoz J, Majcherczyk P, Que YA, Rousson V, Moreillon P, Entenza JM. Use of a human-like lowgrade bacteremia model of experimental endocarditis to study the role of Staphylococcus aureus adhesions and platelet aggregation in early endocarditis. Infect Immun. 2013;81:697-703.

\section{Publisher's note}

Springer Nature remains neutral with regard to jurisdictional claims in published maps and institutional affiliations. 